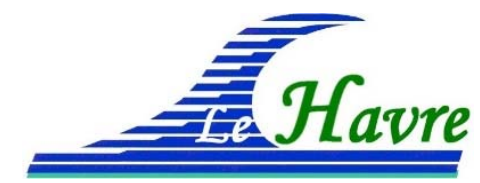

XVI ${ }^{\text {èmes }}$ Journées Nationales Génie Côtier - Génie Civil

Le Havre, 2020

DOI:10.5150/jngcgc.2020.086 C Editions Paralia CFL

disponible en ligne - http://www.paralia.fr - available online

\title{
Evènements de submersion sur la période 1900-2010 à Gâvres (Morbihan) : utilisation conjointe des approches historiques, de modélisation et statistiques
}

\author{
Déborah IDIER ${ }^{1}$, Jérémy ROHMER ${ }^{1}$, Rodrigo PEDREROS ${ }^{1}$, \\ Sylvestre LE ROY ${ }^{2}$, Jérôme LAMBERT ${ }^{1}$, Jessie LOUISOR ${ }^{1}$, \\ Gonéri LE COZANNET ${ }^{1}$, Erwan LE CORNEC ${ }^{3}$
}

\author{
1. BRGM, DRP/R3C, 3 avenue Claude Guillemin, 45060, Orléans, France. \\ d.idier@brgm.fr ; j.rohmer@brgm.fr ; r.pedreros@brgm.fr ; j.louisor@brgm.fr ; \\ g.lecozannet@brgm.fr \\ 2. BRGM, DAT/BRE, 2 rue de Jouanet, 35700 Rennes, France. \\ s.leroy@brgm.fr \\ 3. GEOS-AEL, 12 Rue Maréchal Foch, 56410 Etel, France. \\ geos56@wanadoo.fr
}

\section{Résumé :}

La caractérisation des inondations côtières passées est cruciale pour la prévention des risques. Elle est toutefois limitée par le caractère partiel des informations historiques et par le manque ou la qualité limitée de données hydrométéorologiques passées. Ces questions sont abordées en combinant approches historiques, statistiques et modélisation hydrodynamique. La méthode proposée est appliquée au site de Gâvres (Morbihan), sur la période 1900-2010. Deux bases de données sont constituées : une base de données hydrométéorologiques continue (niveau moyen, marée, surcote, vagues, vent), et une base de données sur les dommages. À l'aide de ces données et d'un modèle hydrodynamique, nous avons identifié 9 événements de submersion, dont 5 significatifs $(1904,1924,1978$, 2001, 2008). L'évènement de submersion du 09/02/1924 est caractérisé par une période de retour bi-variée de dépassement $\operatorname{Tr}$ (niveau d'eau par rapport au niveau moyen ; hauteur significative des vagues) supérieure à 1000 ans. L'étude de l'évolution à long terme du risque d'inondation, en évaluant comment $T r$ varie avec la remontée du niveau marin, montre que Tr atteint des valeurs inférieures à 1 an, pour 8 des 9 événements historiques de submersion, pour une élévation du niveau de la mer de 0,63 m.

Mots-clés : Historique, Statistiques d'extrêmes bi-variés, Modélisation hydrodynamique, SWASH, Franchissement par paquets de mer, Submersion.

\section{Introduction}

La caractérisation (occurrence, mécanismes, probabilité) des inondations côtières passées est cruciale pour la prévention des risques, mais rencontre un certain nombre de difficultés (surtout si l'on considère les événements qui se sont produits il y a plusieurs décennies). La plupart du temps, les informations historiques sont très partielles, avec par exemple la 


\section{Thème 7 - Risques côtiers}

mention de l'eau envahissant des quais ou des dommages subis par un bien donné. Même partielles, ces informations restent très utiles pour améliorer la quantification des niveaux d'eau extrêmes, pour mieux estimer les risques potentiels d'inondation, mieux identifier les zones exposées, ou mieux comprendre les impacts du changement climatique contemporain (BULTEAU et al., 2015 ; GILOY et al., 2019). Toutefois, les informations historiques sur les inondations sont sujettes à des incertitudes : si l'on est très confiant dans la réalité des inondations signalées, l'absence d'information ne signifie pas nécessairement qu'il n'y a pas eu d'inondation. C'est notamment le cas sur les sites côtiers qui n'avaient que peu ou pas d'enjeux jusqu'aux dernières décennies, et donc où il est possible que personne n'ait signalé l'inondation ou n'avait de raisons de le faire. En outre, la connaissance limitée des conditions hydrométéorologiques passées met à mal notre capacité à identifier les conditions hydrométéorologiques critiques, qui peuvent conduire à des inondations. Ces connaissances sont cruciales non seulement pour les systèmes d'alerte, mais aussi pour anticiper l'effet potentiel de l'élévation du niveau de la mer. Cette étude vise à montrer comment la connaissance de l'occurrence des événements passés et de leurs conditions de forçage (et probabilité associée) peut être améliorée en combinant approches historiques, statistiques et modélisation numérique. Nous nous concentrons sur le site macrotidal de Gâvres (Morbihan), et étudions la période 1900-2010. Le site, la méthode et le modèle de submersion sont décrits dans la section 2. La section 3 décrit les résultats principaux en termes d'évènements de submersions passées, des conditions hydrométéorologiques associées ainsi que de leur occurrence (et l'effet de la remontée du niveau marin sur cette dernière).

\section{Site, méthodologie et modèle}

\subsection{La presqu'île de Gâvres}

La presqu'île de Gâvres est située sur la côte atlantique métropolitaine, en milieu macrotidal (figure 1). Ce site a notamment été touché par la tempête Johanna du 10 mars 2008 (figure 2 ; voir aussi LE ROY et al., 2015), qui a provoqué l'inondation d'environ 120 maisons (Maire de Gâvres : communication personnelle). Lors de cette tempête, la submersion a principalement été induite par le franchissement des défenses côtières par les vagues. Les processus d'inondation à Gâvres sont connus pour être complexes (influence des marées ; rôle prépondérant des franchissements par paquets de mer; variété des défenses côtières naturelles et humaines, exposition aux vagues contrastée suivant les façades - ex : Goërem et Grande Plage -). En outre, ce territoire a connu de fortes évolutions (morphologie, occupation du territoire, ... ; voir (IDIER et al., 2020)). 


\section{XVİ̀mes Journées Nationales Génie Côtier - Génie Civil \\ Le Havre 2020}
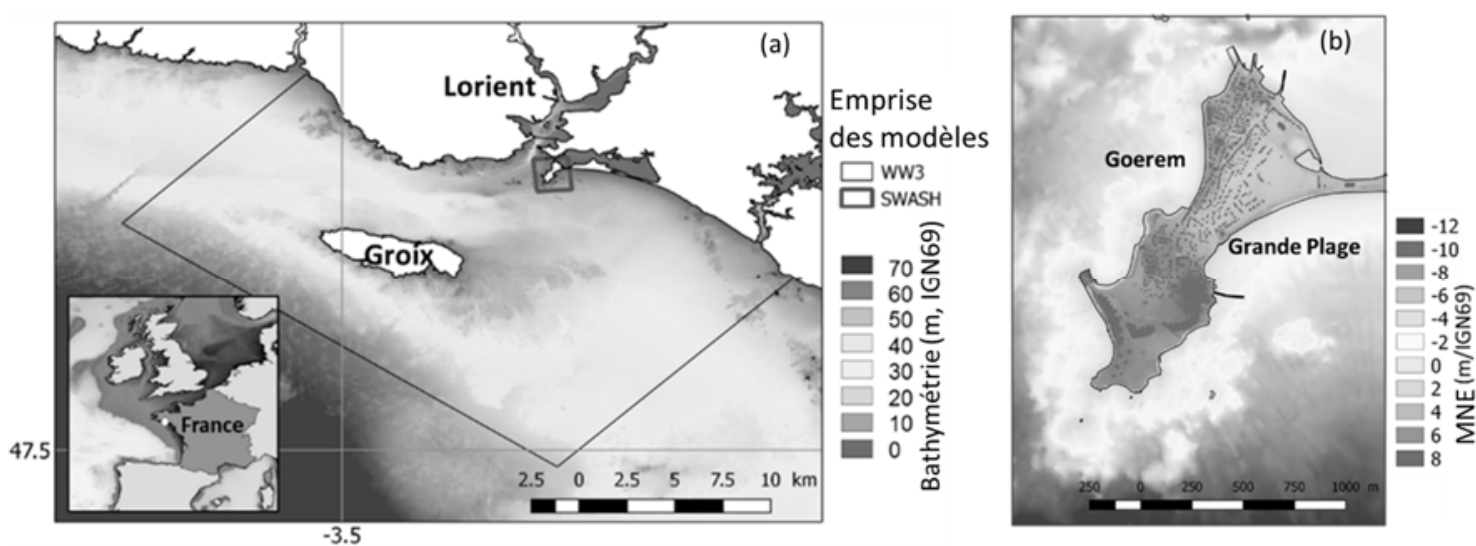

Figure 1. Site d'étude : (a) localisation et emprise des modèles, (b), topo-bathymétrie (Elévation, IGN69).

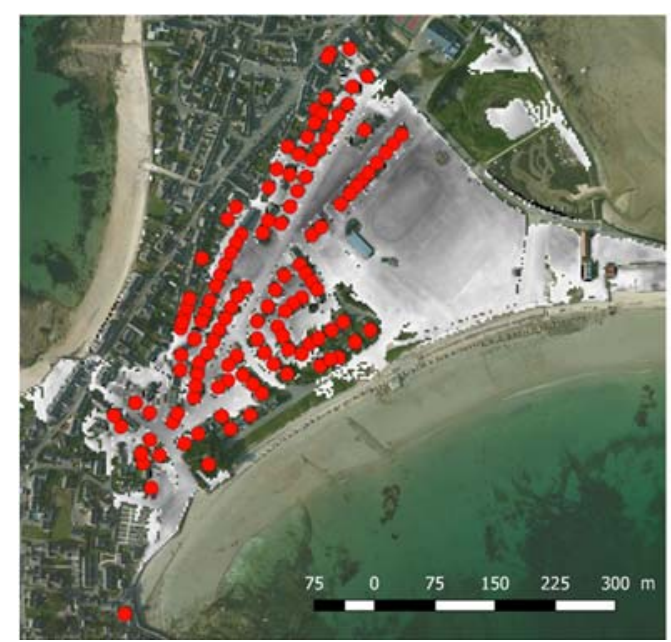

\section{Observations}

- Maisons inondées

Résultats modèle $(H \max (\mathrm{m}))$

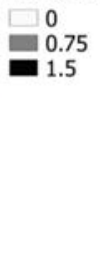

Figure 2. Evènement de submersion du 10/03/2008 (Johanna) : observations et hauteur d'eau maximale simulée avec le modèle hydrodynamique.

\subsection{Méthode}

La méthode consiste à tout d'abord créer une base de données de dommages, basée sur les études antérieures, la presse (ancienne et contemporaine), et les archives municipales, départementales et militaires. Nous sommes repartis de travaux de LE CORNEC et al. (2012) et avons complété la base de données à partir de presse ancienne. Dans cette base de données Dommages, les évènements identifiés comme évènements de submersion sont classés en terme d'intensité (0: pas de submersion, 1: submersion modérée, 2: submersion significative), associée à un indice de confiance. On définit comme évènement de submersion tout évènement tel qu'une partie (même faible) du territoire a été recouvert d'eau. Parallèlement, une base de données hydrométéorologiques continues de 1900 à 2010 a été construite, intégrant : niveau moyen (relatif, en IGN69), marée (locale), surcote atmosphérique (locale), vagues (au large, Sud de Groix) et vent (dans l'emprise WW3, figure 1a). De par leur localisation géographique (IDIER et al., 2020), 


\section{Thème 7 - Risques côtiers}

ces données ne sont pas sensibles aux évolutions bathymétriques sur la période d'étude. Cette base de données a été construite en utilisant des simulations rétrospectives de différentes couvertures temporelles, en corrigeant celles de moins bonne qualité par celles de meilleure qualité, via une méthode de correction quantile-quantile. Les différentes bases de données utilisées sont : 20CR, CFSR, MARC, Sonel-vagues-20CR, BoBWA, Homere, Norgasug. Pour chacun des évènements de dommages (submersion ou non), les conditions hydrométéorologiques sont ensuite extraites de la base de données et utilisées en entrée d'un modèle de submersion (section 2.3). Pour chaque évènement de dommage le modèle fournit une information complémentaire sur l'existence ou pas d'un évènement de submersion. Ces 3 bases de données sont alors confrontées. En cas d'incohérence, des recherches historiques complémentaires sont effectuées. A la fin, la base de données Dommages est mise à jour en termes d'identification des évènements de submersion, de classe d'intensité, et d'indices de confiance associés.

\subsection{Modèle de submersion}

La modélisation est basée sur le modèle à résolution de phase non hydrostatique SWASH (ZIJLEMA et al., 2011), car il permet non seulement de simuler le débordement et les écoulements à terre, mais surtout le franchissement par paquets de mer, qui est joue un rôle primordial sur notre site d'étude. La mise en œuvre et la validation du modèle pour le site de Gâvres sont décrites dans IDIER et al. (2020). Ici, nous rappelons les éléments principaux. Le domaine de calcul est indiqué sur la figure 1a. Les résolutions spatiale et temporelle sont respectivement de $3 \mathrm{~m}$ en horizontal, de 2 couches sur la verticale et de plus de $10 \mathrm{~Hz}$. La topo-bathymétrie utilisée est représentative de mars 2008. Les conditions de vagues en mer (au sud de l'île de Groix) sont propagées jusqu'aux limites du modèle SWASH en utilisant le modèle spectral de vagues WW3 (ARDHUIN et al., 2010); voir le grand domaine spatial sur la figure 1a. La mise en place de ces modèles a été validée en comparant les zones inondées modélisées et observées, pour l'événement Johanna (figure 2) : le modèle surestime légèrement le nombre de maisons inondées d'environ $3 \%$, ce qui peut être considéré comme un résultat très satisfaisant pour des environnements aussi complexes. Nous utilisons ce modèle pour estimer un indicateur d'occurrence et intensité de la submersion. Comme indicateur, nous avons choisi le volume d'eau entrant dans les terres ( $\mathrm{Vol}$ ) à marée haute, sur un laps de temps de 15 minutes. Une telle simulation coûte $1 \mathrm{~h} 30 \mathrm{~min}$ de temps de calcul (sur 48 cœurs). Il convient de garder à l'esprit que la topo-bathymétrie a évolué de manière significative au cours du temps et que cette modélisation, faite pour une topo-bathymétrie fixée (2008) ne vise pas à reproduire exactement les submersions passées. 


\section{XVİ̀mes Journées Nationales Génie Côtier - Génie Civil \\ Le Havre 2020}

\section{Résultats}

3.1 Evènements historiques de submersion

La confrontation des bases de données et des résultats du modèle pour chaque évènement de la base de données Dommages a mené à rechercher des informations historiques complémentaires sur plusieurs évènements dont celui du 02/02/1904. 9 évènements historiques de submersion sont identifiés sur la période 1900-2010, dont 5 correspondant à des submersions significatives (tableau 1). Parmi ces 5, seul l'évènement de 1904 a un indice de confiance faible. En effet, contrairement aux 4 autres évènements, les éléments historiques trouvés pour cet évènement indiquent des dommages sans mention explicite d'une submersion massive (une submersion importante est toutefois attestée dans de nombreux secteurs environnants : Lorient, Groix, Quiberon, Belle-Ile...), alors que d'après le modèle, ce serait l'évènement pour lequel on aurait la plus forte valeur d'indicateur de submersion Vol (tableau 1). Les conditions hydrométéorologiques associées à ces 9 évènements de submersion sont indiquées dans le tableau 1 (les évènements significatifs étant en gras).

Tableau 1. Caractéristiques hydrométéorologiques à pleine-mer (PM) pour les 9 évènements de submersion identifiés à Gâvres entre 1900 et 2010 : niveau moyen de la mer par rapport au 0 IGN69 (MSL), marée (T), surcote (S), hauteur de vagues significative (Hs), période pic des vagues (Tp), direction pic des vagues (Dp), vitesse du vent (U), Direction du vent (DU). En gras : évènements de submersion significative (1ère colonne), valeur maximale (autres colonnes).

\begin{tabular}{|l|l|l|l|l|l|l|l|l|l|l|}
\hline Date & $\begin{array}{l}P M \\
(\mathrm{TU})\end{array}$ & $\begin{array}{l}\mathrm{MSL} \\
(\mathrm{m})\end{array}$ & $\begin{array}{l}\mathrm{T}(\mathrm{m}) \\
(\mathrm{m})\end{array}$ & $\begin{array}{l}\mathrm{S} \\
(\mathrm{m})\end{array}$ & $\begin{array}{l}\mathrm{Tp} \\
(\mathrm{s})\end{array}$ & $\begin{array}{l}\mathrm{Dp} \\
\left({ }^{\circ} \mathrm{N}\right)\end{array}$ & $\begin{array}{l}U \\
(\mathrm{~m} / \mathrm{s})\end{array}$ & $\begin{array}{l}\mathrm{DU} \\
\left({ }^{\circ} \mathrm{N}\right)\end{array}$ & $\begin{array}{l}\mathrm{Vol} \\
\left(\mathrm{m}^{3}\right)\end{array}$ \\
\hline $\mathbf{0 2 / 0 2 / 1 9 0 4}$ & $04: 00$ & 0.307 & 2.438 & 0.48 & 5.71 & 15.1 & 247.6 & 14.6 & 224.4 & 7803 \\
\hline $\mathbf{0 9 / 0 1 / 1 9 2 4}$ & $05: 30$ & 0.362 & 2.128 & $\mathbf{0 . 6 5}$ & $\mathbf{8 . 4 9}$ & $\mathbf{2 1 . 3}$ & 258.5 & 7.8 & 310.8 & 4514 \\
\hline $\mathbf{2 6 / 0 2 / 1 9 7 8}$ & $05: 30$ & 0.451 & 2.058 & 0.55 & 5.61 & 18.8 & 242.0 & 10.5 & 209.7 & 3548 \\
\hline $\mathbf{1 0 / 0 1 / 2 0 0 1}$ & $03: 40$ & 0.499 & 2.238 & 0.24 & 3.49 & 11.2 & 232.6 & 13.3 & 224.7 & 32 \\
\hline $07 / 02 / 2001$ & $15: 20$ & 0.499 & 1.988 & 0.28 & 4.16 & 15.7 & 251.3 & 3.9 & 137.4 & 15 \\
\hline $27 / 10 / 2004$ & $15: 20$ & 0.508 & 1.978 & 0.35 & 4.42 & 9.23 & 199.9 & 13.6 & 199.6 & 0.6 \\
\hline $\mathbf{1 0 / 0 3 / 2 0 0 8}$ & $05: 20$ & 0.515 & 2.358 & 0.55 & 5.33 & 11.0 & 255.7 & 18.2 & 264.1 & 1987 \\
\hline $10 / 02 / 2009$ & $04: 00$ & 0.518 & $\mathbf{2 . 3 9 8}$ & 0.07 & 5.93 & 13.2 & 248.0 & 13.4 & 299.6 & 163 \\
\hline $28 / 02 / 2010$ & $03: 10$ & $\mathbf{0 . 5 2 1}$ & 2.348 & 0.60 & 2.57 & 9.0 & 189.7 & $\mathbf{1 8 . 4}$ & 301.7 & 312 \\
\hline
\end{tabular}

Dans cette étude, nous considérons 8 conditions de forçage (niveau moyen de la mer, marée, houle, hauteur des vagues, période des vagues, direction des vagues, intensité du vent et direction du vent). Ce problème à 8 dimensions peut être réduit à un problème à 6 dimensions en remplaçant les trois premières composantes par le niveau de l'eau au repos résultant $(\xi)$. Les 5 principales inondations correspondent à des combinaisons de 


\section{Thème 7 - Risques côtiers}

paramètres différentes. Les événements de 1924 et 1978 sont caractérisés par un niveau d'eau $\xi$ élevé ( 3,14 et 3,06 m IGN69), des vagues hautes ( $\mathrm{Hs}=8,5$ et 5,6 m) et longues $(\mathrm{T} p=21,2$ et $18,8 \mathrm{~s})$, et des vents modérés $(\mathrm{U}=7,2$ et $10,5 \mathrm{~m} / \mathrm{s})$ de $\mathrm{NO}$ et $\mathrm{SO}$. L'inondation de 2001 se caractérise par un niveau d'eau calme plus bas (2,97 m IGN69), des vagues plus petites $(\mathrm{Hs}=3,5 \mathrm{~m})$ et plus courtes $(\mathrm{Tp}=11,2 \mathrm{~s})$, et des vents plus forts $(13,5 \mathrm{~m} / \mathrm{s}) \mathrm{du}$ sud-ouest, mais nous devons garder à l'esprit la rupture d'ouvrages de défense le long de la plage sud (Grande Plage) lors de cette submersion. L'inondation de 2008 est caractérisée par un niveau d'eau $\xi$ beaucoup plus haut (3,42 m IGN69), une forte hauteur de vague $(\mathrm{Hs}=5,53 \mathrm{~m})$ et une période modérée $(\mathrm{Tp}=11 \mathrm{~s})$, pour des vents encore plus forts $(18,2 \mathrm{~m} / \mathrm{s})$ venant de l'ouest. Les caractéristiques de l'événement de 1904 semblent similaires à celles de 2008 (fort niveau d'eau, forte hauteur de vague) mais avec une période de vague plus grande $(\mathrm{T} p=15,1 \mathrm{~s})$. L'événement de dommages associé au niveau d'eau $\xi$ le plus élevé ( $\xi=3.47$ m IGN69) correspond à la tempête Xynthia de 2010, mais cet événement est identifié comme une inondation modérée, puisque seul un peu de franchissement a été observé. Les conditions hydrométéorologiques correspondantes sont assez similaires à celles de l'inondation de 2008, mais avec une hauteur de vague deux fois plus faible. Cela suggère (et est confirmé par les résultats du modèle de submersion (IDIER et al., 2020)) que sans les opérations de rehaussement des ouvrages de défense suite à la submersion de 2008, la tempête Xynthia aurait entraîné une inondation plus importante.

\subsection{Probabilité d'occurrence}

En 110 ans, au moins 4 inondations importantes ont eu lieu. Cela suggère une période de retour empirique des inondations significatives sur la zone de Gâvres d'environ 25 ans. En considérant l'évènement de submersion de 1904, 20 ans pourrait être considérée comme une limite inférieure pour la période de retour des submersions significatives. Il s'agit d'une estimation empirique qui doit être considérée avec prudence. Cependant, la topographie et les défenses côtières ont fortement évolué au cours du siècle, entre autres après l'événement de Johanna, qui a conduit à augmenter la hauteur des défenses côtières. En outre, aucune estimation fiable de la période de retour des submersions (qu'elles soient importantes ou non) ne peut être fournie à l'aide de la présente base de données, étant donné que des événements avec une submersion modérée n'ont probablement pas été identifiés (dans les recherches de documents historiques) au cours de la première moitié du siècle (voir IDIER et al., 2020).

Cependant, en utilisant la base de données hydrométéorologiques, continue nous pouvons caractériser la probabilité d'occurrence des conditions hydrométéorologiques qui ont conduit, dans le passé, à des inondations importantes ou modérées. À cette fin, une analyse bivariée des valeurs extrêmes (bEVA) est effectuée en se concentrant sur le niveau de l'eau au repos (marée, surcote atmosphérique) par rapport au niveau moyen de la mer $\left(\xi_{\mathrm{MSL}}=\xi-\mathrm{MSL}\right)$ et la hauteur des vagues $(\mathrm{Hs})$, qui sont les principaux facteurs 


\section{XVIèmes Journées Nationales Génie Côtier - Génie Civil \\ Le Havre 2020}

menant à la submersion sur ce site (IDIER et al., 2020). Rappelons ici que le niveau moyen (MSL) varie avec le temps et est donc différent pour chaque évènement. Le principe de la méthode bEVA est d'extrapoler la densité de probabilité conjointe des variables de niveau d'eau et hauteur de vagues ( $\xi_{\mathrm{MSL}}$; Hs) à des valeurs extrêmes en tenant compte de la structure de dépendance. En utilisant la base de données hydrométéorologique, nous suivons une procédure similaire à celle décrite par NICOLAE et al. (2018) ; pour plus de détails pour le cas présent, voir (IDIER et al., 2020). Au final, nous obtenons une période Tr de dépassement conjoint (figure 3). Parmi les 5 évènements de submersions significatives, les conditions ( $\xi_{\mathrm{MSL}}$; Hs) ayant mené à la submersion du 10 janvier 2001 sont caractérisées par une période de retour très petite $(<1 \mathrm{an})$, suggérant un rôle important des défaillances de défense côtière qui ont eu lieu pendant cet événement. La période de retour la plus importante ( $>1000$ ans) est observée lors de l'événement de 1924, tandis que $\operatorname{Tr} \in$ [100-200] ans et $\operatorname{Tr} \approx 100$ ans pour les événements de 1904 et 2008, respectivement. On peut également noter que les conditions de l'événement de 2010 en mer ont eu une période de retour $(\operatorname{Tr} \approx 20$ ans) plus longue que celle de 1978 ( $\operatorname{Tr} \approx 10$ ans $)$.

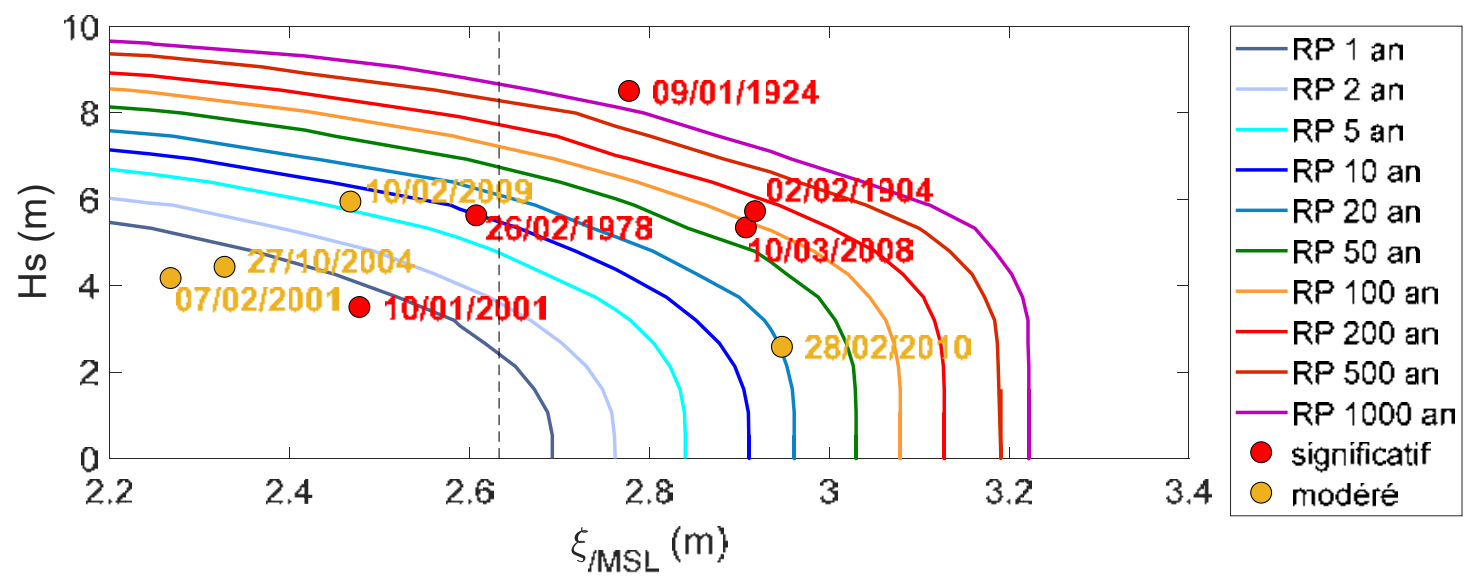

Figure 3. Contours de dépassement conjoints dans l'espace ( $\xi_{\mathrm{MSL}}$; Hs) pour les valeurs de la période de retour allant de 10 à 1000 ans et positionnement des neuf événements historiques. Notez que le niveau de l'eau calme $\xi_{\mathrm{MSL}}$ est exprimé par rapport au niveau moyen de la mer, c'est-à-dire $\xi_{\mathrm{MSL}}=\xi$ - MSL. Les événements d'inondation de la base

de données des dommages sont indiqués par des marqueurs de couleur.

\subsection{Effets de la remontée du niveau marin}

On peut alors discuter de l'impact de l'élévation du niveau de la mer sur les périodes de retour Tr. Par exemple, en supposant que le niveau de l'eau $\left(\xi_{1904}\right)$ de l'événement de 1904 soit atteint en 2017 , la contribution correspondante de la marée et de la surcote ( $\left.\xi_{\mathrm{MSL}}\right)$ serait plus faible, et Tr serait diminuée d'un facteur supérieur à 5 (pour l'événement de $1904: \xi_{\operatorname{MSL}(2017)}=20$ ans, à comparer à 100 ans $<\xi_{\text {MSL(1904) }}<200$ ans). Cette analyse est 


\section{Thème 7 - Risques côtiers}

étendue à une large gamme de valeurs d'élévation du niveau de la mer. La figure 4 montre les variations de $\operatorname{Tr}$ en fonction de l'élévation moyenne du niveau de la mer (nommé SLR), en prenant pour référence le niveau moyen de 2017 ( $S L R=0)$, pour chacun des évènements de submersion significative. Pour tous les événements, sauf celui de 1924, $\mathrm{Tr}$ diminue jusqu'à des valeurs inférieures à 1 an pour $\mathrm{SLR} \leq 0,63 \mathrm{~m}$. Cette valeur de SLR est égale à la remontée moyenne régionale médiane du niveau de la mer à l'horizon 2100 fournie par le 5ieme rapport d'évaluation du GIEC pour le scénario RCP8.5, d'après les données fournies par le centre de données climatiques intégrées de l'Université de Hambourg, disponibles en ligne : http://icdc.cen.uni-hamburg.de/daten/ocean/ar5slr.html (CARSON et al., 2016). Cela suggère que d'ici 2100, les conditions conjointes ( $\xi$; Hs) correspondant aux inondations passées se produiraient statistiquement au moins une fois par an.

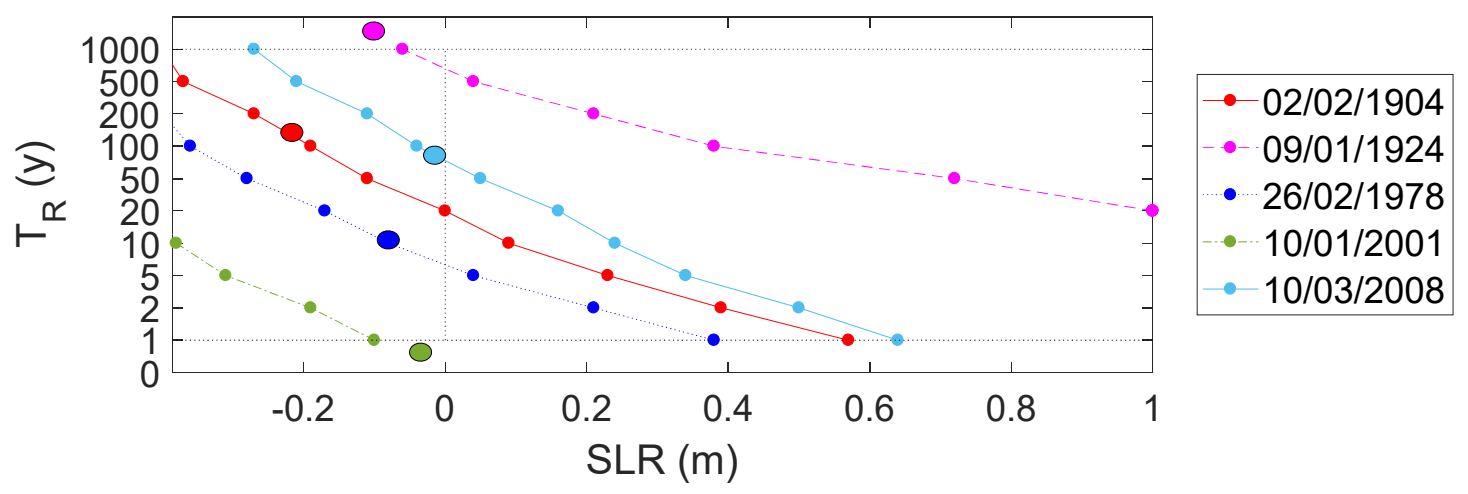

Figure 4. Périodes de retour (Tr) de dépassement conjoint pour les 5 submersions significatives en fonction de l'élévation moyenne du niveau de la mer (SLR). SLR=0 correspond à l'année 2017. Grands cercles : période de retour Tr correspondant aux événements passés lors de leur survenance (i.e. pour le niveau moyen aux dates concernées).

\section{Références bibliographiques}

ARDHUIN F., ROGERS W.E., BABANIN A.V., FILIPOT J., MAGNE R., ROLAND A., VAN DER WESTHUYSEN A., QUEFFEULOU P., LEFEVRE J., AOUF L., COLLARD F. (2010). Semi empirical dissipation source functions for ocean waves. Part I: Definition, calibration, and validation. J. Phys. Oceanogr., Vol. 40, pp 1917- 1941. https://doi.org/10.1175/2010JPO4324.1

BULTEAU T., IDIER D., LAMBERT J., GARCIN M. (2015). How historical information can improve estimation and prediction of extreme coastal water levels: application to the Xynthia event at La Rochelle (France). Nat. Hazards Earth Syst. Sci., Vol. 15, pp 1135-1147. https://doi.org/10.5194/nhess-15-1135-2015 


\section{XVIèmes Journées Nationales Génie Côtier - Génie Civil Le Havre 2020}

CARSON M., KÖHL A., STAMMER D., SLANGEN A.B.A., KATSMAN C.A., VAN DE WAL R.S.W., CHURCH J., WHITE N. (2016). Coastal sea level changes, observed and projected during the 20th and 21st century. Climatic Change, Vol. 134(1-2), pp 269281. https://doi.org/10.1007/s10584-015-1520-1

GILOY N., HAMDI Y., BARDET L., GARNIER E., DULUC C. M. (2019) Quantifying historic skew surges: an example for the Dunkirk Area, France. Natural Hazards, Vol. 98, pp 869-893. https://doi.org/10.1007/s11069-018-3527-1

IDIER D, ROHMER J, PEDREROS R, LE ROY S, LAMBERT J, LOUISOR J, LE COZANNET G, LE CORNEC E. (2020). Coastal flood: a composite method for past events characterisation providing insights in past, present and future hazards. Joining historical, statistical and modeling approaches. Natural Hazards, in press. https://doi.org/10.1007/s11069-020-03882-4

NICOLAE-LERMA A., BULTEAU T., ELINEAU S., PARIS F., DURAND P., ANSELM B., PEDREROS R. (2018). High-resolution marine flood modelling coupling overflow and overtopping processes: framing the hazard based on historical and statistical approaches. Nat Hazards Earth Syst. Sci., Vol. 18, pp 207-229, https://doi.org/10.5194/nhess-18-207-2018

LE CORNEC E., LE BRIS E., VAN LIERDE M. (2012). Atlas des risques littoraux sur le département du Morbihan. Phase 1 : Recensement et conséquences des tempêtes et coups de vent majeurs. Rapport d'étude GEOS-DHI. Direction Départementales des Territoires et de la Mer du Morbihan, $476 \mathrm{p}$.

LE ROY S., PEDREROS R., ANDRE C., PARIS F., LECACHEUX S., MARCHE F., VINCHON C. (2015). Coastal flooding of urban areas by overtopping: dynamic modelling application to the Johanna storm (2008) in Gâvres (France). Nat. Hazards Earth Syst. Sci., Vol. 15, pp 2497-2510. https://doi.org/10.5194/nhess-15-2497-2015

ZIJLEMA M., STELLING G., SMIT P. (2011). SWASH: An operational public domain code for simulating wave fields and rapidly varied flows in coastal waters. Coast. Eng. Vol. 58, pp 992-1012. https://doi.org/10.1016/j.coastaleng.2011.05.015 
Thème 7 - Risques côtiers 